\title{
ERRATUM
}

\section{Functional regulation of the apurinic/apyrimidinic endonuclease 1 by nucleophosmin: impact on tumor biology}

\author{
C Vascotto ${ }^{1}$, L Lirussi ${ }^{1}$, M Poletto ${ }^{1}$, M Tiribelli ${ }^{2}$, D Damiani ${ }^{2}$, D Fabbro ${ }^{1}$, G Damante ${ }^{1}$, B Demple ${ }^{3}$, E Colombo ${ }^{4,5}$ and G Tell ${ }^{1}$
}

${ }^{1}$ Department of Medical and Biological Sciences, University of Udine, Udine, Italy; ${ }^{2}$ Department of Experimental and Clinical Medical Sciences, University of Udine, Udine, Italy; ${ }^{3}$ Department of Pharmacological Sciences, Stony Brook University School of Medicine, Stony Brook, NY, USA; ${ }^{4}$ Department of Experimental Oncology, European Institute of Oncology, Milan, Italy and ${ }^{5}$ Dipartimento di Scienze della Salute, Universita' degli Studi di Milano, Milan, Italy

Oncogene (2015) 34, 3482; doi:10.1038/onc.2015.191

Correction to: Oncogene (2014) 33, 2876-2887; doi: 10.1038/onc. 2013.251; published online 8 July 2013

Since the publication of the above article, it has been noted that the affiliation for E Colombo was incorrect. The correct affiliation is given above.
The publisher apologises for any inconvenience caused by this error. 